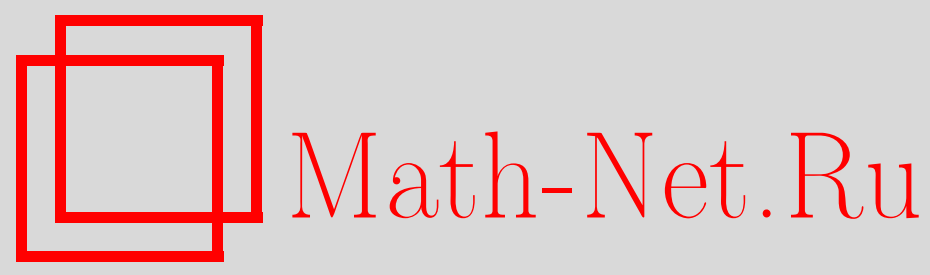

В. В. Филиппов, О спектре Фучика и периодических решениях, Матем. заметки, 2003, том 73, выпуск 6, 910-922

DOI: https://doi.org/10.4213/mzm228

Использование Общероссийского математического портала Math-Net.Ru подразумевает, что вы прочитали и согласны с пользовательским соглашением http://www. mathnet.ru/rus/agreement

Параметры загрузки:

IP : 54.209 .52 .79

26 апреля 2023 г., 08:52:16

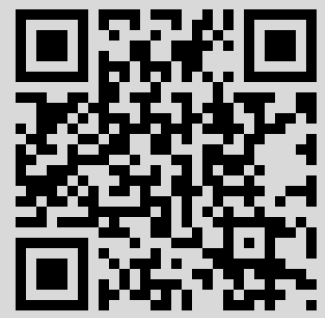




\title{
О СПЕКТРЕ ФУЧИКА И ПЕРИОДИЧЕСКИХ РЕШЕНИЯХ
}

\section{В. В. Филиппов}

\begin{abstract}
Продолжается распространение теории краевых задач на обыкновенные дифференциальные уравнения и включения с разрьвными правыми частями, основанное на построении нового варианта метода сдвига вдоль траекторий. В заметке новый подход сопоставляется с результатами, использующими спектр Фучика.
\end{abstract}

Библиография: 15 названий.

Настоящая статья посвящена дальнейшей разработке варианта [1]-[3] метода сдвига вдоль траекторий, приспособленного, в частности, к работе с уравнениями и включениями с разрывными правыми частями.

В этой статье мы воспользуемся ситуациями, связанными со спектром Фучика, для введения новых элементов в метод [1]-[3], в частности, идеологии, наработанной в [4]-[7] в рамках использования метода Лерэ-Шаудера (см. [4]-[7], где можно найти дальнейшие ссылки, а также [8]).

Учитывая, что основная часть нашего изложения ведется на уровне аксиоматической теории [9]-[12], наши результаты ввиду развитой теории задачи Коши применимы в очень широких предположениях.

Как правило, терминологию и обозначения теории [9]-[12] мы будем использовать без дополнительных обсуждений.

1. Оценка асимптотических свойств пространства решений. Априорные оценки периодических решений уравнения $x^{\prime \prime}+g(x)=h(t)$ получают обычно, исходя из асимптотики функции $g$, см. [4]-[7].

В [13] был предложен подход к получению априорных оценок, основанный на предельном переходе в пространстве пространств решений $R_{\mathrm{ceh}}(U)$, когда оценивается не асимптотика членов уравнения, а асимптотика строения пространств решений. Исследуя по этой схеме уравнение $x^{\prime}=f(t, x)$, рассматриваем семейство замен переменнњх $(t, x) \rightarrow(t, \mu x)$ и оцениваем возможньй предел (если он существует, он определен не однозначно, из всех возможных берем самьй удобньй) пространств решений уравнений $x^{\prime}=\mu f(t, x / \mu)$ при $\mu \rightarrow 0$ в пространстве пространств решений $R_{\text {ceh }}(U)$.

Пусть, например, оказьвается, что при $\mu \rightarrow 0$ правые части уравнений $x^{\prime}=\mu f(t, x / \mu)$ равномерно на компактах сходятся к правой части уравнения $x^{\prime}=f_{0}(t, x)$. Мы можем рассмотреть гомотопию правой части, определив ее по формуле

$$
F(t, x, \mu)= \begin{cases}\mu f(t, x / \mu) & \text { при } \mu \in(0,1], \\ f_{0}(t, x) & \text { при } \mu=0 .\end{cases}
$$

Работа выполнена при поддержке Российского фонда фундаментальных исследований, грант № 01-01-00744. 
К этой гомотопии мы можем применять принцип продолжения по параметру. То есть в асимптотику правой части уже заложено использование вполне определенной гомотопии, и нет необходимости ради применения принципа продолжения по параметру подыскивать еще какую-то. Правда, ситуация, когда правые части уравнений $x^{\prime}=\mu f(t, x / \mu)$ сходятся к правой части некоторого уравнения $x^{\prime}=f_{0}(t, x)$, достаточно редка. Но довольно обычна ситуация, когда правые части уравнений $x^{\prime}=\mu f(t, x / \mu)$ сходятся в смысле полунепрерывности сверху к правой части некоторого дифференциального включения $x^{\prime} \in F_{0}(t, x)$, свойства решений которого легко устанавливаются. Тогда опять мы можем рассмотреть гомотопию (теперь многозначную) правой части, определив ее по формуле

$$
F(t, x, \mu)= \begin{cases}\mu f(t, x / \mu) & \text { при } \mu \in(0,1], \\ F_{0}(t, x) & \text { при } \mu=0 .\end{cases}
$$

К этой гомотопии мы тоже можем применять принцип продолжения по параметру. Как и в предыдущем случае, в асимптотику правой части уже заложено использование вполне определенной гомотопии и нет необходимости ради применения принципа продолжения по параметру подыскивать еще какую-то. Этот случай уже оказывается достаточно общим.

Более того, обычно указьвается асимптотика слагаемых уравнения. На самом деле, эта асимптотика обычно лишь указывает фигурировавшее в предыдущем абзаце предельное включение. Поэтому нет необходимости проявлять чудеса изобретательности, получая априорные оценки и готовя тем самым использование принципа продолжения по параметру, т.е. можно пользоваться уже заложенной в асимптотику гомотопией предыдущего абзаца.

На самом же деле, нет необходимости и рассматривать гомотопию. Вместо условия сходимости при $\mu \rightarrow 0$ достаточно предположить сходимость соответствующих пространств решений для некоторой последовательности $\mu_{i} \rightarrow 0$ к пространству решений дифференциального включения $x^{\prime} \in F_{0}(t, x)$. При достаточно больших $i$ топологические свойства пространства решений уравнения $x^{\prime}=\mu_{i} f\left(t, x / \mu_{i}\right)$ в контексте рассматриваемых вопросов оказываются аналогичньми свойствам пространства решений дифоференциального включения $x^{\prime} \in F_{0}(t, x)$. Поэтому принцип продолжения по параметру достаточно применить к гомотопии, когда $\mu$ меняется на отрезке $\left[\mu_{i}, 1\right]$. Но учитьвая вполне конкретную связь уравнения $x^{\prime}=\mu f(t, x / \mu)$ с исходньм, можно вообще обойтись без принципа продолжения по параметру. Это было использовано уже в [14].

Сказанное не покрывает все случаи, рассмотренные в [4]-[6]. Дело в том, что в [4]-[6] естественный кандидат на предельное дифференциальное включение $x^{\prime} \in F_{0}(t, x)$ может обладать ненужными периодическими решениями. Поэтому мы не можем отследить требуемые степени и изложенная выше схема перестает работать.

Габдрахманов [8] увидел, что в предположениях [4]-[6], подобрав нужным образом последовательность $\mu_{i} \rightarrow 0$, можно сузить дифференциальное включение $x^{\prime} \in F_{0}(t, x)$ до некоторого включения $x^{\prime} \in G(t, x)$ (т.е. $G(t, x) \subseteq F_{0}(t, x)$ ), которое уже не обладает соответствующими периодическими решениями, но по-прежнему пространства решений уравнений $x^{\prime}=\mu_{i} f\left(t, x / \mu_{i}\right), \mu_{i} \rightarrow 0$, сходятся к пространству решений дифференциального включения $x^{\prime} \in G(t, x)$. Мы дадим новую интерпретацию наблюдению Габдрахманова.

Пространство $C_{s}^{+}\left(U, t_{0}\right)$ максимально продолженных вправо от точки $t_{0}$ функций описано в [1]. 
Множество $M \subseteq C_{s}^{+}\left(U, t_{0}\right)$ назовем ограниченным на отрезке $\left[t_{0}, t_{1}\right]$, если найдется такое число $r$, что $\left\|\left.z\right|_{\left[t_{0}, t_{1}\right] \cap \pi(z)}\right\| \leqslant r$. Ниже, когда $a<0<T<b$ и $U=(a, b) \times \mathbb{R}^{n}$, все функции из ограниченного на $[0, T]$ множества определены на всем отрезке $[0, T]$.

TEOPEMA $1.1 .^{1}$ Пусmb

1) $a<0<T<b, U=(a, b) \times \mathbb{R}^{n}, r>0 u W$ - открытое ограниченное на отрезке $[0, T]$ подмножество пространства $C_{s}^{+}(U, 0)$;

2) $Y, Y_{0} \in R_{\mathrm{ceh}}(U), Y_{0} \subseteq Y u$, если $z \in Y^{+} \cap \partial W u z(0)=z(T)$, mo $\left.z\right|_{I} \notin Y_{0} \partial л я$ любого невырохденного отрезка $I \subseteq[0, T]$;

3) $\left\{Z_{i}: i=1,2, \ldots\right\} \subseteq R_{\mathrm{ceh}}(U), Z_{i} \rightarrow Y$ при $i \rightarrow \infty$, и пространство

$$
\left(\lim _{i \rightarrow \infty} \operatorname{sop} \sup Z_{i}\right) \cap \partial W
$$

не содержит функиий $z$, удовлетворяющих условию $z(0)=z(T)$.

Тогда, начиная с некоторого $i=i_{0}$, выполнены условия

а) пространство $Z_{i} \cap \partial W$ не содержит функиий $z$, удовлетворяющ,хх условию $z(0)=z(T)$

b) степень сдвига $z \rightarrow z(T)-z(0)$ пересечения $Z_{i} \cap \partial W, i=i_{0}, i_{0}+1, \ldots$, совпадает со степенью сдвига пересечения $Y_{0} \cap \partial W$.

ДокаЗАтельство. І. Для $\lambda \in[0, T]$ и $i=1,2, \ldots$ положим $Z_{i, \lambda}=Y_{0} *_{\lambda} Z_{i}$.

Здесь $Z *_{s} Y$ обозначает результат подклеивания пространства $Y$ к пространству $Z$, причем в качестве вспомогательной функции $h$ берется проектирование произведения $\mathbb{R} \times \mathbb{R}^{n}$ на сомножитель $\mathbb{R}$, т.е. пространство $Z *_{s} Y$ при $t \leqslant s$ совпадает с пространством $Z$, а при $t \geqslant s$ совпадает с пространством $Y$, см. [10, $\S$ VIII.7] или [11, 11.5$]$.

Имеем $\left(Z_{i, 0}\right)_{[0, T] \times \mathbb{R}^{n}}=\left(Z_{i}\right)_{[0, T] \times \mathbb{R}^{n}}$ и $\left(Z_{i, T}\right)_{[0, T] \times \mathbb{R}^{n}}=\left(Y_{0}\right)_{[0, T] \times \mathbb{R}^{n}}$.

II. Покажем, что найдется такое число $i_{0}=1,2, \ldots$, что для любого $i=i_{0}, i_{0}+1, \ldots$ ни при каком $\lambda \in[0, T]$ задача $z \in Z_{i, \lambda}^{+} \cap \partial W, z(0)=z(T)$ не имеет решений.

Допустив противное, получаем существование таких чисел $\lambda_{j} \in[0, T], j=1,2, \ldots$, и функций $z_{j} \in Z_{i_{j}, \lambda_{j}}^{+} \cap \partial W$, что $z_{j}(0)=z_{j}(T)$ и $i_{1}<i_{2}<\cdots$.

Переходя, если нужно, к подпоследовательности, добиваемся в силу компактности отрезка $[0, T]$ вьполнения дополнительного условия: последовательность $\left\{\lambda_{j}\right.$ : $j=1,2, \ldots\}$ сходится к некоторому $\Lambda \in[0, T]$.

Из I и условия 3 ) следует, что $\Lambda \neq 0$, так как в случае $\Lambda=0$ в силу сходимости $Z_{i_{j}, \lambda_{j}} \rightarrow \lim$ top $\sup _{i \rightarrow \infty} Z_{i}$ (теорема VIII.7.1 в [10] или теорема 11.5 .1 в [12]) получаем, что множество $\left(\lim\right.$ top $\left.\sup _{i \rightarrow \infty} Z_{i}\right) \cap \partial W$ содержит функцию $z$, для которой $z(0)=z(T)$ (предельную для функций $z_{j}, j=1,2, \ldots$ ), что противоречит условию 3 ).

Если же $\Lambda \neq 0$, то в силу сходимости $Z_{i_{j}, \lambda_{j}} \rightarrow Y_{0} *_{\Lambda} Y$ (теорема VIII.7.1 в [10] или теорема 11.5.1 в [12]) получаем аналогичное противоречие с условием 2).

III. Полагая в II $\lambda=0$, получаем вьполнение условия а). Требуемое в условии b) совпадение степеней следует, например, из леммы 2.7 в [3] или из результатов [2] при рассмотрении гомотопии $\lambda \rightarrow Z_{i, \lambda}$. Теорема доказана.

\footnotetext{
${ }^{1}$ Назовем ее принципом Габдрахманова.
} 
2. О сходимости последовательностей пространств решений. Дифференциальное включение

$$
x^{\prime \prime} \in F\left(t, x, x^{\prime}\right)
$$

считаем равносильным системе

$$
\left\{\begin{array}{l}
x^{\prime}=y \\
y^{\prime} \in F(t, x, y)
\end{array}\right.
$$

или векторному уравнению

$$
u^{\prime} \in F_{0}(t, u)
$$

где

$$
u=\left(\begin{array}{l}
x \\
y
\end{array}\right), \quad F_{0}(t, u)=\left(\begin{array}{c}
y \\
F(t, x, y)
\end{array}\right) .
$$

Именно систему (2.2) или уравнение (2.3) мы будем иметь в виду, говоря о пространстве решений включения второго порядка (2.1). Это пространство решений будем обозначать через $D^{\prime}(F)$. То есть $D^{\prime}(F)=D\left(F_{0}\right)$.

Основным элементом при нашем подходе к теории задачи Коши [9]-[12] является умение доказывать сходимость последовательностей пространств решений.

Пусть

$$
\begin{gathered}
-\infty \leqslant a<b \leqslant \infty,-\infty \leqslant c<d \leqslant \infty, U=(a, b) \times((c, d) \times \mathbb{R}), \\
F: U \rightarrow \mathbb{R}-\text { многозначное отображение; }
\end{gathered}
$$

$F_{i}: U \rightarrow \mathbb{R}, \quad i=1,2, \ldots$, - последовательность многозначных отображений. (2.5)

K анализу положения вещей вне множества

$$
E=(a, b) \times((c, d) \times\{0\})
$$

применимы методы общей теории [9]-[12]. Специального рассмотрения требует только анализ ситуации около множества $E$. В этом пункте мы внесем добавления в замечания, сделанные на эту тему в [13]. Обозначим

$$
V=U \backslash E \text {. }
$$

\section{Рассмотрим}

УТВЕРЖДЕНИЕ 2.1. Пусть в обозначениях (2.4)-(2.7) выполнены следующие условия:

1) $D^{\prime}\left(F_{i}, V\right) \rightarrow D^{\prime}(F, V)$;

2) если $(t, x, 0) \in U$, mо $F(t, x, 0) \neq \varnothing ;$ если при әтом inf $F(t, x, 0) \leqslant 0 u$ $\sup F(t, x, 0) \geqslant 0$, mo $0 \in F(t, x, 0)$

3) если $(t, x, 0) \in U u \inf F(t, x, 0)>0$, то найдутся такое число $\varepsilon>0 u$ такие интегрируемые функиии $\varphi_{i}:[t-\varepsilon, t+\varepsilon] \rightarrow \mathbb{R}, i=1,2, \ldots$, что $(t-\varepsilon, t+\varepsilon) \times(x-\varepsilon, x+\varepsilon) \times(-\varepsilon, \varepsilon) \subseteq U, \inf F_{i}(s, u, v) \geqslant \varphi_{i}(s)$ nри всеx $(s, u, v) \in(t-\varepsilon, t+\varepsilon) \times(x-\varepsilon, x+\varepsilon) \times(-\varepsilon, \varepsilon) u i=1,2, \ldots$, последовательность функиий

$$
\Phi_{i}(\xi)=\int_{t}^{\xi} \varphi_{i}(s) d s, \quad i=1,2, \ldots,
$$

равномерно сходится $к$ монотонно возрастающей функиии $\Phi$; 
4) если $(t, x, 0) \in U u \sup F(t, x, 0)<0$, то найдутся такое число $\varepsilon>0$ $u$ такие интегрируемые функиии $\varphi_{i}:[t-\varepsilon, t+\varepsilon] \rightarrow \mathbb{R}, i=1,2, \ldots$, что $(t-\varepsilon, t+\varepsilon) \times(x-\varepsilon, x+\varepsilon) \times(-\varepsilon, \varepsilon) \subseteq U, \sup F_{i}(s, u, v) \leqslant-\varphi_{i}(s)$ nри всех $(s, u, v) \in(t-\varepsilon, t+\varepsilon) \times(x-\varepsilon, x+\varepsilon) \times(-\varepsilon, \varepsilon) u i=1,2, \ldots$, и последовательность функиий

$$
\Phi_{i}(\xi)=\int_{t}^{\xi} \varphi_{i}(s) d s, \quad i=1,2, \ldots,
$$

равномерно сходится $к$ монотонно возрастающей функции $\Phi$.

Тогда $D^{\prime}\left(F_{i}\right) \rightarrow D^{\prime}(F)$.

ДокАЗАТЕЛЬСТво. І. Начнем с обсуждения выполнения условия равностепенной непрерьвности

$$
\left\{D^{\prime}\left(F_{i}\right): i=1,2, \ldots\right\} \in s(U) .
$$

Здесь ситуация не отличается от разобранной в [13].

Пусть $K$ - произвольное компактное подмножество области $U$. Рассмотрим проектирование $\varphi$ компакта $K$ на плоскость $\mathbb{R} \times \mathbb{R}$ переменных $t$ и $x$. Абсолютные значения ординат точек компакта $K$ ограничены сверху некоторым числом $m>0$. Так как для любого решения $z=\left(x_{z}, y_{z}\right)$ любого из рассматриваемых включений выполнена оценка $\left|x_{z}^{\prime}(t)\right|=\left|y_{z}(t)\right| \leqslant m$, то

семейство функций $\left\{x_{z}: z \in \bigcup\left\{D^{\prime}\left(F_{i}, K\right): i=1,2, \ldots,\right\}\right\}$ равностепенно непрерывно.

В силу утверждения 1.1 из [13] для любой точки $\left(t_{0}, x_{0}, y_{0}\right) \in K$ множество

$$
\begin{array}{r}
P=\bigcap\left\{\left\lfloor\left\{\operatorname{Im} z: z \in Z_{j}, j=k, k+1, \ldots, \pi(z) \subseteq\left(t_{0}-\varepsilon, t_{0}+\varepsilon\right),\right.\right.\right. \\
\left.\left.\left.\operatorname{Im} z \cap O_{\delta} x_{0} \neq \varnothing\right\}\right]: k=1,2, \ldots, \varepsilon>0, \delta>0\right\}
\end{array}
$$

лежит $^{2}$ в множестве $\varphi^{-1} \varphi\left(t_{0}, x_{0}, y_{0}\right)$. В силу специального вида отображения $\varphi$ (оно определено как проектирование) множество $\varphi^{-1} \varphi\left(t_{0}, x_{0}, y_{0}\right)$ есть пересечение компакта $K$ с прямой $x=x_{0}, t=t_{0}$, т.е. $\varphi^{-1} \varphi\left(t_{0}, x_{0}, y_{0}\right)=\varphi^{-1}\left(t_{0}, x_{0}\right) \cap K$.

При фиксированном $t_{0} \in \mathbb{R}$ это множество пересекается с множеством $E_{t_{0}}$ всего лишь по одной точке $\left(t_{0}, x_{0}, 0\right)$, поэтому мы находимся в сфере действия следствия теоремы VI.3.1 в [10] (= следствие 1 теоремы 7.3 .1 в [12]), что и дает вьполнение условия равностепенной непрерьвности $\left\{D^{\prime}\left(F_{i}\right): i=1,2, \ldots\right\} \in s(U)$.

II. Пусть теперь $(t, x, 0) \in U, \varepsilon>0, W=(t-\varepsilon, t+\varepsilon) \times(x-\varepsilon, x+\varepsilon) \times(-\varepsilon, \varepsilon) \subseteq U$ и интегрируемые функции $\varphi_{i}:[t-\varepsilon, t+\varepsilon] \rightarrow \mathbb{R}, i=1,2, \ldots$, таковы, что

$$
\inf F_{i}(s, u, v) \geqslant \varphi_{i}(s)
$$

при всех $(s, u, v) \in(t-\varepsilon, t+\varepsilon) \times(x-\varepsilon, x+\varepsilon) \times(-\varepsilon, \varepsilon)$ и $i=1,2, \ldots$,

\footnotetext{
${ }^{2}$ На самом деле, из (2.8) легко вытекает, что множества

$$
\begin{aligned}
\bigcap\left\{\bigcup \left\{\operatorname{Im}(x): z=(x, y) \in Z_{j}, j=k, k+1, \ldots, \pi(z) \subseteq\left(t_{0}-\varepsilon, t_{0}+\varepsilon\right),\right.\right. \\
\left.\left.\left.\qquad \operatorname{Im}(x) \cap O_{\delta} x_{0} \neq \varnothing\right\}\right]: k=1,2, \ldots, \varepsilon>0, \delta>0\right\}
\end{aligned}
$$
}

одноточечны, что и дает требуемое. 
и последовательность функций

$$
\Phi_{i}(\xi)=\int_{t}^{\xi} \varphi_{i}(s) d s, \quad i=1,2, \ldots
$$

равномерно сходится к монотонно возрастающей функции $\Phi$.

Если $z \in \lim$ top $\sup _{i \rightarrow \infty} D^{\prime}\left(F_{i}, W\right)$, то существует последовательность $z_{j}=$ $\left(x_{j}, y_{j}\right) \in D^{\prime}\left(F_{i_{j}}, W\right), i_{1}<i_{2}<\cdots$, сходящаяся к $z$.

Если точки $s<t$ лежат внутри отрезка $\pi(z)$, то, начиная с некоторого $j=j_{0}$, эти точки $s<t$ лежат и внутри отрезка $\pi\left(z_{j}\right)$.

Имеем

$$
\begin{gathered}
y(t)-y(s)=\lim _{j \rightarrow \infty}\left(y_{j}(t)-y_{j}(s)\right) \\
y_{j}(t)-y_{j}(s)=\int_{s}^{t} y_{j}^{\prime}(\xi) d \xi \geqslant \int_{s}^{t} \varphi_{j}(\xi) d \xi=\Phi_{j}(t)-\Phi_{j}(s) \rightarrow \Phi(t)-\Phi(s) .
\end{gathered}
$$

Сопоставляя это с (2.10), получаем $y(t)-y(s) \geqslant \Phi(t)-\Phi(s)>0$, а это означает, что график функции $z$ может иметь не более одной общей точки с множеством $E$. Таким образом, мы находимся в предположениях $\S \S$ VI.4 и VI.5 в [10] (= $\S 7.4$ и 7.5 в [12]). В силу сделанных там замечаний $D^{\prime}\left(F_{i}, W\right) \rightarrow D^{\prime}(F, W)$.

Случай, когда вместо условия (2.9) выполнено условие

$$
\begin{gathered}
\sup F_{i}(s, u, v) \leqslant-\varphi_{i}(s) \\
\text { при всех }(s, u, v) \in(t-\varepsilon, t+\varepsilon) \times(x-\varepsilon, x+\varepsilon) \times(-\varepsilon, \varepsilon) \text { и } i=1,2, \ldots,
\end{gathered}
$$

рассматривается аналогично.

III. Пусть $\gamma$ - семейство всех множеств $W$ вида, указанного в II. В силу условий 2)-4) для любой точки $\left(t_{0}, x_{0}, 0\right)$ замкнутого множества $E_{0}=E \backslash \bigcup \gamma$ мы имеем $0 \in F\left(t_{0}, x_{0}, 0\right)$.

IV. Завершающая часть рассуждения повторяет конец доказательства утверждения 2.1 в [13].

Рассмотрим произвольную функцию $z=(x, y) \in \lim \sup _{i \rightarrow \infty} D^{\prime}\left(F_{i}\right)$. Пусть

$$
M=\left\{t \in \pi(z):(t, z(t)) \in E_{0}\right\} .
$$

В силу условия 1$)$ и II вне множества $M$ функция $x$ является решением включения $x^{\prime \prime} \in F\left(t, x, x^{\prime}\right)$. Нам остается проверить, что

$$
x^{\prime \prime}(t) \in F\left(t, x(t), x^{\prime}(t)\right) \text { при почти всех } t \in M \text {. }
$$

Для этого заметим, что $\left.y\right|_{M} \equiv 0$, и поэтому

$$
y^{\prime}(t)=0 \text { при почти всех } t \in M \text {. }
$$

Но в силу III $0 \in F(t, x(t), 0)$, поэтому выполнение условия (2.13) означает вьполнение (2.12).

Таким образом, $z \in D^{\prime}(F)$, что и требовалось. Утверждение доказано.

Полагая $F_{i} \equiv F$, получаем 
СЛЕДСТВИЕ. Пусть выполнены условия (2.4), (2.6), (2.7), условие 2) из утверждения 2.1, а также следующие условия:

1) $D^{\prime}(F, V) \in R_{\mathrm{ce}}(V)$;

2) если $(t, x, 0) \in U$ u inf $F(t, x, 0)>0$, то найдутся такое число $\varepsilon>0$ и такая интегрируемая почти всюду положительная функиия $\varphi:[t-\varepsilon, t+\varepsilon] \rightarrow \mathbb{R}$, что $(t-\varepsilon, t+\varepsilon) \times(x-\varepsilon, x+\varepsilon) \times(-\varepsilon, \varepsilon) \subseteq U u \inf F(s, u, v) \geqslant \varphi(s)$ nри всех $(s, u, v) \in(t-\varepsilon, t+\varepsilon) \times(x-\varepsilon, x+\varepsilon) \times(-\varepsilon, \varepsilon)$

3) если $(t, x, 0) \in U u \sup F(t, x, 0)<0$, то найдутся такое число $\varepsilon>0$ и такая интегрируемая почти всюду полохительная функиия $\varphi:[t-\varepsilon, t+\varepsilon] \rightarrow \mathbb{R}$, что $(t-\varepsilon, t+\varepsilon) \times(x-\varepsilon, x+\varepsilon) \times(-\varepsilon, \varepsilon) \subseteq U u \sup F_{i}(s, u, v) \leqslant-\varphi_{i}(s) n p u$ вcex $(s, u, v) \in(t-\varepsilon, t+\varepsilon) \times(x-\varepsilon, x+\varepsilon) \times(-\varepsilon, \varepsilon)$.

Тогда $D^{\prime}(F) \in R_{\mathrm{c}}(U)$.

То есть в этих предположениях решения включения $x^{\prime \prime} \in F\left(t, x, x^{\prime}\right)$ непрерывно зависят от начальных условий.

В соответствии с общим подходом (9)-(12) утверждения типа утверждения 2.1 плодотворно используются для доказательства существования решения задачи Коши для уравнений соответствующих типов. Но это уже выходит за рамки целей этой публикации, и эти возможности мы оставляем без рассмотрений; см. также [13], где рассматривались более узкие условия.

3. Спектр Фучика. В качестве примера приложения теоремы 1.1 воспроизведем рассуждение Габдрахманова из [8], внеся в него соответствующие изменения. В [8], как и в [4]-[6] для оценки асимптотики членов уравнения используется спектр Фучика.

$C$ пктр Фучика - это подмножество $S$ первой четверти евклидовой плоскости, которое определяется как объединение

$$
S=\bigcup\left\{C_{m}: m \in \mathbb{N}\right\}, \quad \text { где } C_{m}=\left\{(\mu, \nu): \frac{1}{\sqrt{\mu}}+\frac{1}{\sqrt{\nu}}=\frac{2}{m}\right\} .
$$

Роль спектра Фучика в связи с задачей о существовании периодических решений периода $2 \pi$ можно проиллюстрировать следующим образом. Рассмотрим две пары чисел $0<\alpha_{1} \leqslant \beta_{1}$ и $0<\alpha_{2} \leqslant \beta_{2}$ и обозначим через $I_{\alpha_{1}, \beta_{1} ; \alpha_{2}, \beta_{2}}(x)$ отрезок $\left[\alpha_{1}, \beta_{1}\right]$ для $x \geqslant 0$ и отрезок $\left[\alpha_{2}, \beta_{2}\right]$ для $x<0$.

Рассмотрим включение $x^{\prime \prime} \in-I(x) x$ со следующими свойствами:

$$
\begin{gathered}
I(x)=I_{\alpha_{1}, \beta_{1} ; \alpha_{2}, \beta_{2}}(x), \text { где } 0<\alpha_{1} \leqslant \beta_{1}, 0<\alpha_{2} \leqslant \beta_{2} ; \\
\text { точка }\left(\alpha_{1}, \alpha_{2}\right) \text { лежит на ветви } C_{m} \text { спектра } \Phi \text { уика, } \\
\text { а точка }\left(\beta_{1}, \beta_{2}\right) \text { лежит на ветви } C_{m+1}, \text { где } m=1,2, \ldots,
\end{gathered}
$$

т.е.

$$
\frac{1}{\sqrt{\alpha_{1}}}+\frac{1}{\sqrt{\alpha_{2}}}=\frac{2}{m}, \quad \frac{1}{\sqrt{\beta_{1}}}+\frac{1}{\sqrt{\beta_{2}}}=\frac{2}{m+1} .
$$

Как мы договорились в п. 3 , дифференциальное включение $x^{\prime \prime} \in-I(x) x$ при исследовании заменяется соответствуюшей системой первого порядка, пространство $Z$ решений которого принадлежит классу $R_{\mathrm{ceh}}\left(\mathbb{R} \times \mathbb{R}^{2}\right)$ и является коническим (т.е. инвариантно относительно замен переменных $(t, x) \rightarrow(t, \lambda x))$. Поэтому максимально продолженные решения этого включения определены на всей действительной прямой и, если решение в какой-то точке отлично от $\overrightarrow{0}$, то оно уже нигде не обращается в $\overrightarrow{0}$, см. [2] и [15]. 
ЗАмечАниЕ 3.1. Допустим, что в предположениях (3.1), (3.2) сушествует ненулевое периодическое решение $x(t)$ включения $x^{\prime \prime} \in-I(x) x$ периода $2 \pi$. Нетрудно проверить, что функция $x(t)$ есть либо решение уравнения $x^{\prime \prime}+g_{\alpha_{1}, \alpha_{2}}(x) x=0 \mathrm{c}$

$$
g_{\alpha_{1}, \alpha_{2}}(x)= \begin{cases}\alpha_{1} & \text { при } x \geqslant 0, \\ \alpha_{2} & \text { при } x<0,\end{cases}
$$

либо решение уравнения $x^{\prime \prime}+g_{\beta_{1}, \beta_{2}}(x) x=0$.

ЗАМЕЧАНИЕ 3.2. Пусть $m<\gamma<m+1, m=1,2, \ldots$, и

$W$ - ограниченная окрестность тождественного нуля в пространстве $C_{s}^{+}(U, 0)$.

Уравнение $x^{\prime \prime}+g_{\gamma, \gamma}(x) x=0$ имеет вид линейного уравнения $x^{\prime \prime}+\gamma x=0$. Нетрудно проверить, что уравнение $x^{\prime \prime}+g_{\gamma, \gamma}(x) x=0$ не имеет ненулевых $2 \pi$-периодических решений. Поэтому в силу линейности этого уравнения степень сдвига ${ }^{3}$ границы $\partial W$ вдоль траекторий уравнения $x^{\prime \prime}+\gamma x=0$ на временном отрезке длиной $2 \pi$ отлична от нуля.

ЗАмечАниЕ 3.3. Рассмотрим любую пару чисел $\alpha_{1}$ и $\alpha_{2}$ такую, что точка $\left(\alpha_{1}, \alpha_{2}\right)$ лежит между ветвями $C_{m}$ и $C_{m+1}$ спектра Фучика, т.е.

$$
\frac{2}{m+1}<\frac{1}{\sqrt{\alpha_{1}}}+\frac{1}{\sqrt{\alpha_{2}}}<\frac{2}{m} \text {. }
$$

Заметим, что мы можем включить параметры $\alpha_{1}$ и $\alpha_{2}$ в семейства $\beta_{1}(t)$ и $\beta_{2}(t), 0 \leqslant t \leqslant 1$, таким образом, что $\beta_{1}(1)=\alpha_{1}, \beta_{2}(1)=\alpha_{2}, \beta_{1}(0)=\beta_{2}(0)$ и

$$
\frac{2}{m+1}<\frac{1}{\sqrt{\beta_{1}(t)}}+\frac{1}{\sqrt{\beta_{2}(t)}}<\frac{2}{m}
$$

при всех $0 \leqslant t \leqslant 1$. В силу замечания 3.2 и сохранения степени при гомотопии, см. [2] или [3], мы получаем, что в обозначениях (3.3) степень сдвига границы $\partial W$ вдоль траекторий уравнения $x^{\prime \prime}+g_{\alpha_{1}, \alpha_{2}}(x) x=0$ отлична от нуля.

Обозначая в предположениях (3.1), (3.2) через $Y$ пространство решений включения $x^{\prime \prime} \in-I(x) x$, через $Y_{0}$ пространство решений уравнения

$$
x^{\prime \prime}+g_{\left(\alpha_{1}+\beta_{1}\right) / 2,\left(\alpha_{2}+\beta_{2}\right) / 2}(x) x=0,
$$

мы попадаем в силу сделанных замечаний в предположения теоремы 1.1. Из нее вьводим

Теорема 3.1. Пусть $Z \in R_{\mathrm{ceh}}(U)$, пространство $Z_{\lambda}$ получается из пространства $Z$ при замене переменны $(t, x) \rightarrow(t, \lambda x), \lambda_{i} \rightarrow 0$, в обозначениях (3.1), (3.2) последовательность $Z_{\lambda_{i}}$ при $i \rightarrow \infty$ сходится $\kappa$ пространству решений включения $x^{\prime \prime} \in-I(x) x$ и в обозначениях (3.3) пространство (lim top $\left.\sup _{i \rightarrow \infty} Z_{\lambda_{i}}\right) \cap \partial W$ не содержит решений $z$, удовлетворяющих условию $z(0)=z(T)$. Тогда найдется такая функиия $z \in Z^{+}$, что $z(0)=z(T)$.

В [6] доказана следующая теорема, подводящая итог развитию этой тематики, см. также [8].

\footnotetext{
${ }^{3}$ Здесь и в других аналогичных ситуациях под сдвигом границы $\partial W$ вдоль решений из $Y_{1}$ понимается отображение $z \rightarrow z(0)-z(T)$ для $z \in \partial W \cap Y_{1}$. Некорректность речи допущена, чтобы вызвать ассоциацию с обычным методом сдвига.
} 
Теорема 3.2. Пусть в обозначения $(3.1)$, (3.2) функиия $g: \mathbb{R} \rightarrow \mathbb{R}$ непрерывна на множестве $\mathbb{R} \backslash\{0\}$, функиия $r: \mathbb{R} \rightarrow \mathbb{R}$ непрерывна, функиия $h:[0,2 \pi] \rightarrow \mathbb{R}$ интегрируема, $\alpha_{1} \leqslant g(x) \leqslant \beta_{1}$ при всех $x>0, \alpha_{2} \leqslant g(x) \leqslant \beta_{2}$ при всех $x<0, g(0)=0$, $\lim _{|x| \rightarrow \infty} r(x) / x=0$. Пусть выполнено по крайней мере одно из следуюших условиนั:

$$
\begin{array}{lll}
\alpha_{1}<\limsup _{x \rightarrow \infty} \frac{1}{x} \int_{0}^{x} g(s) d s & u & \liminf _{x \rightarrow \infty} \frac{1}{x} \int_{0}^{x} g(s) d s<\beta_{1} ; \\
\alpha_{2}<\limsup _{x \rightarrow-\infty} \frac{1}{x} \int_{0}^{x} g(s) d s & u & \liminf _{x \rightarrow-\infty} \frac{1}{x} \int_{0}^{x} g(s) d s<\beta_{2} ; \\
\alpha_{1}<\limsup _{x \rightarrow \infty} \frac{1}{x} \int_{0}^{x} g(s) d s & u & \liminf _{x \rightarrow-\infty} \frac{1}{x} \int_{0}^{x} g(s) d s<\beta_{2} ; \\
\alpha_{2}<\limsup _{x \rightarrow-\infty} \frac{1}{x} \int_{0}^{x} g(s) d s & u & \liminf _{x \rightarrow \infty} \frac{1}{x} \int_{0}^{x} g(s) d s<\beta_{1} .
\end{array}
$$

Тогда задача $x^{\prime \prime}+g(x) x+r(x)=h(t), x(0)=x(2 \pi), x^{\prime}(0)=x^{\prime}(2 \pi)$ имеет решение.

ДокАЗАтЕльство не претендует на оригинальность. Оно опирается, в частности, на идеи, уже использованные в [8] и [6], хотя и содержит новые элементы. В частности, мы ссылаемся на нашу теорему 3.1 .

I. Замена переменных $(t, x) \rightarrow(t, \lambda x)$ переводит уравнение $x^{\prime \prime}+g(x) x+r(x)=h(t)$ в уравнение $x^{\prime \prime}+g(x / \lambda) x+\lambda r(x / \lambda)=\lambda h(t)$. В силу утверждения 2.1 при $\lambda \rightarrow 0$ пространства $Z_{\lambda}$ решений этих уравнений сходятся к пространству решений $Z$ включения $x^{\prime \prime} \in-I(x) x \operatorname{c~} I(x)=I_{\alpha_{1}, \beta_{1} ; \alpha_{2}, \beta_{2}}(x)$.

Для ссылки на теорему 3.1 нам осталось проверить, что пространство

$$
\left(\lim _{i \rightarrow \infty} \operatorname{top} \sup Z_{\lambda_{i}}\right) \cap \partial W
$$

не содержит решений $z$, удовлетворяющих условию $z(0)=z(T)($ с $T=2 \pi)$, для некоторой последовательности $\lambda_{i} \rightarrow 0$ и соответствующим образом подобранного множества $W$.

II. Пусть выполнено условие (3.4). Обозначим через $m$ верхнюю грань значений $\sup \left\{|x(t)|,\left|x^{\prime}(t)\right|: 0 \leqslant t \leqslant 2 \pi\right\}$ по всем решениям $x$ включения $x^{\prime \prime} \in-I(x) x$, удовлетворяющим условиям

$$
\pi(x)=[0,2 \pi], \quad x(0)=x(2 \pi) \leqslant 1, \quad x^{\prime}(0)=x^{\prime}(2 \pi)=0 .
$$

Обозначим через $W$ множество функций $z=(x, y)$ пространства $C_{s}^{+}(U, 0)$, удовлетворяющих условиям

$$
\sup \{x(t): t \in[0,2 \pi] \cap \pi(z)\}<1 \quad \text { и } \sup \{|x(t)|,|y(t)|: t \in[0,2 \pi] \cap \pi(z)\}<2 m \text {. }
$$

Множество $W$ открыто в пространстве $C_{s}^{+}(U, 0)$.

Из условия (3.4) и непрерьвности функции

$$
\Phi(u)=\frac{1}{u} \int_{0}^{u} g(s) d s
$$


вытекает существование такой последовательности $u_{i} \rightarrow \infty$, что предел $\lim _{i \rightarrow \infty} \Phi\left(u_{i}\right)$ существует и лежит строго между числами $\alpha_{1}$ и $\beta_{1}: \alpha_{1}<\lim _{i \rightarrow \infty} \Phi\left(u_{i}\right)<\beta_{1}$. Положим $\lambda_{i}=1 / u_{i}$.

Производные функций

$$
\varphi_{i}(x)=\frac{1}{u_{i}} \int_{0}^{u_{i} x} g(s) d s=\lambda_{i} \int_{0}^{x / \lambda_{i}} g(s) d s=\int_{0}^{x} g\left(\frac{v}{\lambda_{i}}\right) d v
$$

удовлетворяют неравенствам $\alpha_{1} \leqslant\left|\varphi_{i}^{\prime}\right| \leqslant \beta_{1}$ при $x \geqslant 0$ и $\alpha_{2} \leqslant\left|\varphi_{i}^{\prime}\right| \leqslant \beta_{2}$ при $x \leqslant 0$. Поэтому наш выбор последовательности $u_{i} \rightarrow \infty$ мы можем осуществить так, что функции $\varphi_{i}^{\prime}$ слабо сходятся к некоторой функции $\psi$, удовлетворяющей тем же неравенствам. В силу результатов [14] (или главы 13 в [12]) и п. 2 настоящей работы пространства решений уравнений

$$
x^{\prime \prime}+g\left(\frac{x}{\lambda_{i}}\right) x+\lambda_{i} r\left(\frac{x}{\lambda_{i}}\right)=\lambda_{i} h(t)
$$

сходятся к пространству решений уравнения $x^{\prime \prime}+\psi(x) x=0$. При этом

$$
\int_{0}^{1} \psi(s) d s=\lim _{i \rightarrow \infty} \int_{0}^{1} g\left(\frac{v}{\lambda_{i}}\right) d v=\lim _{i \rightarrow \infty} \frac{1}{u_{i}} \int_{0}^{u_{i}} g(s) d s=\lim _{i \rightarrow \infty} \Phi\left(u_{i}\right) .
$$

Таким образом, $\alpha_{1}<\int_{0}^{1} \psi(s) d s<\beta_{1}$. Но тогда в силу замечания 3.1 среди решений уравнения $x^{\prime \prime}+\psi(x) x=0$ нет функций, удовлетворяющих условиям $x(0)=x(2 \pi)$, $x^{\prime}(0)=x^{\prime}(2 \pi)$ и $\sup \{x(t): t \in[0,2 \pi] \cap \pi(z)\}=1^{4}$. Ситуация здесь вполне аналогична рассмотренным в [6]. Поэтому мы можем применить теорему 3.1 , что в данном случае и дает требуемое. В случае (3.4) теорема доказана.

III. Случай вьполнения условия (3.5) рассматривается аналогично.

IV. Пусть вьполнено условие (3.6). Если при этом

$$
\liminf _{x \rightarrow \infty} \frac{1}{x} \int_{0}^{x} g(s) d s<\beta_{1},
$$

то мы попадаем в условия II, что и дает требуемое. Нам остается рассмотреть случай

$$
\liminf _{x \rightarrow \infty} \frac{1}{x} \int_{0}^{x} g(s) d s=\beta_{1} .
$$

Ho

$$
\beta_{1} \geqslant \limsup _{x \rightarrow \infty} \frac{1}{x} \int_{0}^{x} g(s) d s
$$

поэтому (3.8) означает, что

$$
\lim _{x \rightarrow \infty} \frac{1}{x} \int_{0}^{x} g(s) d s=\beta_{1} .
$$

Далее повторяем рассуждения II. Обозначим через $m$ верхнюю грань значений

$$
\sup \left\{|x(t)|,\left|x^{\prime}(t)\right|: 0 \leqslant t \leqslant 2 \pi\right\}
$$

\footnotetext{
${ }^{4}$ Функция $\psi$ выражалась бы явно через решения $x=\cos \left(t \sqrt{\alpha_{1}}+t_{0}\right)$ или $x=\cos \left(t \sqrt{\beta_{1}}+t_{0}\right)$ при $x \geqslant 0$ указанных там уравнений.
} 
по всем решениям $x$ включения $x^{\prime \prime} \in-I(x) x$, удовлетворяющим условиям

$$
\pi(x)=[0,2 \pi], \quad x(0)=x(2 \pi) \geqslant-1, \quad x^{\prime}(0)=x^{\prime}(2 \pi)=0 .
$$

Обозначим через $W$ множество функций $z=(x, y)$ пространства $C_{s}^{+}(U, 0)$, удовлетворяющих условиям

$$
\inf \{x(t): t \in[0,2 \pi] \cap \pi(z)\}>-1 \quad \text { и } \sup \{|x(t)|,|y(t)|: t \in[0,2 \pi] \cap \pi(z)\}<2 m .
$$

Множество $W$ открыто в пространстве $C_{s}^{+}(U, 0)$.

Из второй части условия (3.6) и непрерывности функции

$$
\Phi(u)=\frac{1}{u} \int_{0}^{u} g(s) d s
$$

вытекает существование такой последовательности $u_{i} \rightarrow-\infty$, что предел $\lim _{i \rightarrow \infty} \Phi\left(u_{i}\right)$ существует и строго меньше $\beta_{2}: \lim _{i \rightarrow \infty} \Phi\left(u_{i}\right)<\beta_{2}$. Положим $\lambda_{i}=-1 / u_{i}$.

Производные функций

$$
\varphi_{i}(x)=\frac{1}{-u_{i}} \int_{0}^{-u_{i} x} g(s) d s=\lambda_{i} \int_{0}^{x / \lambda_{i}} g(s) d s=\int_{0}^{x} g\left(\frac{v}{\lambda_{i}}\right) d v
$$

удовлетворяют неравенствам $\alpha_{1} \leqslant\left|\varphi_{i}^{\prime}\right| \leqslant \beta_{1}$ при $x \geqslant 0$ и $\alpha_{2} \leqslant\left|\varphi_{i}^{\prime}\right| \leqslant \beta_{2}$ при $x \leqslant 0$. Поэтому наш выбор последовательности $u_{i} \rightarrow-\infty$ мы можем осуществить так, что функции $\varphi_{i}^{\prime}$ слабо сходятся к некоторой функции $\psi$, удовлетворяющей тем же неравенствам. В силу результатов [14] (или главы 13 в [12]) и п. 2 настоящей работы пространства решений уравнений

$$
x^{\prime \prime}+g\left(\frac{x}{\lambda_{i}}\right) x+\lambda_{i} r\left(\frac{x}{\lambda_{i}}\right)=\lambda_{i} h(t)
$$

сходятся к пространству решений уравнения $x^{\prime \prime}+\psi(x) x=0$. При этом

$$
-\int_{0}^{-1} \psi(s) d s=-\lim _{i \rightarrow \infty} \int_{0}^{-1} g\left(\frac{v}{\lambda_{i}}\right) d v=\lim _{i \rightarrow \infty} \frac{1}{u_{i}} \int_{0}^{u_{i}} g(s) d s=\lim _{i \rightarrow \infty} \Phi\left(u_{i}\right) .
$$

Таким образом, $-\int_{0}^{-1} \psi(s) d s<\beta_{2}$. В силу $(3.9) \int_{0}^{1} \psi(s) d s=\beta_{1}$.

Но тогда в силу замечания 3.1 среди решений уравнения $x^{\prime \prime}+\psi(x) x=0$ нет функций, удовлетворяющих условиям

$$
x(0)=x(2 \pi), \quad x^{\prime}(0)=x^{\prime}(2 \pi) \quad \text { и } \quad \sup \{x(t): t \in[0,2 \pi] \cap \pi(z)\}=1 .
$$

Поэтому мы можем применить теорему 3.1 , что в данном случае и дает требуемое. В случае (3.6) теорема доказана.

V. Случай выполнения условия (3.7) рассматривается аналогично IV. Tеорема доказана.

На самом деле, мы заложили в наш подготовительный материал бо́льшие возможности и поэтому использованные в доказательстве теоремы 3.2 рассуждения могут давать и более сильные результаты. Так, применительно к дифференциальным неравенствам аналог теоремы 3.2 может быть сформулирован следующим образом. 
ТЕОрема 3.3. Пусть в обозначениях (3.1), (3.2) функиии $g_{1}, g_{2}: \mathbb{R} \rightarrow \mathbb{R}$ непрерывны на множсетве $\mathbb{R} \backslash\{0\}$, функиия $r: \mathbb{R} \rightarrow[0, \infty)$ непрерывна, функиия $h:[0,2 \pi] \rightarrow$ $[0, \infty)$ интегрируема, $\alpha_{1} \leqslant g_{1}(x) \leqslant g_{2}(x) \leqslant \beta_{1}$ при всех $x>0, \alpha_{2} \leqslant g_{1}(x) \leqslant g_{2}(x) \leqslant$ $\beta_{2}$ при всех $x<0, g_{1}(0)=g_{2}(0)=0, \lim _{|x| \rightarrow \infty} r(x) / x=0$. Пусть выполнено по крайней мере одно из следующих условий:

$$
\begin{array}{lll}
\alpha_{1}<\limsup _{x \rightarrow \infty} \frac{1}{x} \int_{0}^{x} g_{1}(s) d s & u & \liminf _{x \rightarrow \infty} \frac{1}{x} \int_{0}^{x} g_{2}(s) d s<\beta_{1} ; \\
\alpha_{2}<\limsup _{x \rightarrow-\infty} \frac{1}{x} \int_{0}^{x} g_{1}(s) d s & u & \liminf _{x \rightarrow-\infty} \frac{1}{x} \int_{0}^{x} g_{2}(s) d s<\beta_{2} ; \\
\alpha_{1}<\limsup _{x \rightarrow \infty} \frac{1}{x} \int_{0}^{x} g_{1}(s) d s & u & \liminf _{x \rightarrow-\infty} \frac{1}{x} \int_{0}^{x} g_{2}(s) d s<\beta_{2} ; \\
\alpha_{2}<\limsup _{x \rightarrow-\infty} \frac{1}{x} \int_{0}^{x} g_{1}(s) d s & u & \liminf _{x \rightarrow \infty} \frac{1}{x} \int_{0}^{x} g_{2}(s) d s<\beta_{1} .
\end{array}
$$

Пусть многозначное отображсение $F: U \rightarrow \mathbb{R}$ удовлетворяет условию $F(t, x, y) \subseteq$ $-\left[g_{1}(x), g_{2}(x)\right] x+[-r(x)-h(t), r(x)+h(t)]$ при всех $(t, x, y) \in U$ и пространство решений включения $x^{\prime \prime} \in F\left(t, x, x^{\prime}\right)$ принадлежит классу $R_{\mathrm{ceh}}(U)$. Тогда задача $x^{\prime \prime} \in F\left(t, x, x^{\prime}\right), x(0)=x(2 \pi), x^{\prime}(0)=x^{\prime}(2 \pi)$ имеет решение.

ДокАЗАТЕЛьСТВо повторяет доказательство теоремы 3.2 .

Теорема 3.3 сохраняет содержательность и когда функция $F$ является однозначной, т.е. когда речь идет о дифференциальном уравнении $x^{\prime \prime}+F\left(t, x, x^{\prime}\right)=0$, где функция $F$ может быть и разрьвной.

\section{СПИСОК ЦИТИРОВАННОЙ ЛИТЕРАТУРЫ}

[1] Филиппов В.В.О гомологических свойствах множеств решений обыкновенных диффференциальных уравнений // Матем. сб. 1997. Т. 188. №6. С. 139-160.

[2] Филиппов В.В.Смесь методов Лерэ-Шаудера и Пуанкаре-Андронова в задаче о периодических решениях обыкновенных дифференциальных уравнений // Дифференц. уравнения. 1999. T. 35. № 12. C. 1709-1711.

[3] Filippov V. V. Remarks on periodic solutions of ordinary differential equations // J. Dynamical and Control Systems. 2000. V. 6. № 3. P. 431-451.

[4] Gossez J.-P., Omari P. Nonresonance with respect to the Fučik spectrum for periodic solutions of second order ordinary differential equations // Nonlinear Anal. Th. Methods Appl. 1990. V. 14. P. 1079-1104.

[5] Gossez J.-P., Omari P. Periodic solutions of a second order ordinary differential equation: a necessary and sufficient condition for nonresonance // J. of Differential Equations. 1991. V. 94. № 1. P. 67-82.

[6] Habets P., Omari P., Zanolin F. Nonresonance conditions on the potential with respect to the Fučik spectrum for the boundary value problem // Rocky Mountain J. Math. 1995. V. 25. № 4. P. 1305-1340.

[7] Mawhin J. Continuation theorems and periodic solutions of ordinary differential equations // Topological Methods in Differential Equations and Inclusions. Dordrecht-Boston-London: Kluwer Academic Publishers, 1995. P. 291-376.

[8] Габдрахманов С. Р. Пространства решений и спектр Фучика // Дифференц. уравнения. 2003. T. 39. № 3. С. 291-298.

[9] Филиппов В.В. Топологическое строение пространств решений обыкновенных диффференциальных уравнений // УМН. 1993. Т. 48. № 1. С. 103-154.

[10] Филиппов В.В.Пространства решений обыкновенных дифференциальных уравнений. М.: Изд-во МГУ, 1993. 
[11] Filippov V. V. Basic topological structures of the theory of ordinary differential equations // Topology in Nonlinear Analysis. V. 35. Warsaw: Banach Center Publications, 1996. P. 171-192.

[12] Filippov V. V. Basic Topological Structures of Ordinary Differential Equations. Dordrecht-Boston-London: Kluwer Academic Publishers, 1998.

[13] Филиппов В.В.О дифференциальных включениях второго порядка // Фундаментальная и прикладная матем. 1997. Т. 3. №2. С. 587-623.

[14] Филиппов В. В. О существовании периодических решений // Матем. заметки. 1997. Т. 61. № 5. C. $769-784$.

[15] Филиппов В. В. О теореме единственности решения задачи Коши для обыкновенного дифоференциального уравнения // Дифференц. уравнения. 1994. Т. 30. №6. С. 1005-1009.

Московский государственный университет им. М.В. Ломоносова

Поступило

E-mail: vvfil@mech.math.msu.su 